\title{
Humoral Immunity to Stress Proteins and Periodontal Disease
}

\author{
Dennis E. Lopatin, ${ }^{*}$ Charles E. Shelburne, ${ }^{\dagger}$ Neal Van Poperin, * Charles J. Kowalski, * \\ and Robert A. Bagramian ${ }^{\ddagger}$
}

Background: There is evidence that microbial heat shock (stress) proteins (Hsp) are immunodominant antigens of many microorganisms. Immunity to these proteins has been shown in non-oral infections to contribute to protection. This study was undertaken to assess the relationship(s) between immunity to human and microbial heat shock proteins, periodontal disease status, and colonization by periodontal disease-associated microorganisms.

Methods: Subgingival plaque and blood samples obtained from 198 patients during an earlier clinical study were examined for the presence of specific periodontal disease-associated microorganisms and antibodies to selected human and microbial heat shock proteins (Hsp70, Hsp90, DnaK, and GroEL). Particle concentration immunofluorescence assay (PCFIA) was used to detect anti-Hsp antibodies and slot immunoblot assay (SIB) was used to detect subgingival plaque species. Regression models were used to examine the contribution of age, gender, gingival index, probing depth, attachment loss, calculus index, plaque index, and microbial colonization to the anti-Hsp antibody concentrations.

Results: Our studies demonstrated that, when evaluated by ANOVA, patients with higher anti-Hsp (Hsp90, DnaK, and GroEL) antibody concentrations tended to have significantly $(P \leq 0.05)$ healthier periodontal tissues. This was most obvious when the relationship between mean probing depths and antibody concentrations were studied. For Hsp90 antibodies, 2 variables (probing depth and $P$. gingivalis concentration) were found to have significant contributions ( $R=0.293, P<0.0002)$. The equation derived from the regression model was $y=12558-2070 * P D$ $+1842 *$ PG. This confirmed the inverse relationship with probing depth and the positive relationship with colonization by $P$. gingivalis. Attempts to model the other stress protein antibodies were not successful.

Conclusions: We believe that the present observations reflect the presence of protective anti-Hsp antibodies, rather than simply the presence of the microorganism in the gingival sulcus. The clinical significance of these observations lies in the potential of identifying patients who are at risk for developing periodontal disease based on their inability to mount an immune response to specific Hsp or Hsp epitopes, as well as the development of vaccines based on Hsp epitopes. J Periodontol 1999;70:1185-1193.

\section{KEYWORDS}

Periodontal diseases/microbiology; protein, microbial heat-shock; protein, stress; antibody formation; immunity; humoral.

\footnotetext{
* Department of Biologic and Materials Sciences, School of Dentistry, University of Michigan, Ann Arbor, MI. $\uparrow$ M , BioMaterials Technology Center, St. Paul, MN.

† Department of Periodontics, Prevention, and Geriatrics, University of Michigan, Ann Arbor, MI.
}

ssociations between host
response and severity or
progression of periodontal disease have classically been studied in the context of immunity directed against specific microorganisms. ${ }^{1}$ This has been true even though dental plaque represents a highly complex array of microorganisms, each having both unique and common antigens that contribute to its overall antigenicity and immunogenicity. Studying the potential role of host immunity in the context of such a complex antigen mixture is difficult and one is never certain whether differences in host response represent changes in magnitude of the response to a specific antigen or in qualitative differences in the antigenic array. Recently, there has been a greater focus among investigators upon specific purified bacterial antigens such as lipopolysaccharides, lipoteichoic acids, fimbrial proteins, and other cell membrane-associated antigens. ${ }^{2-6}$

Heat shock proteins (Hsp) have been extensively evaluated in numerous medically significant diseases such as candidiasis, Lyme disease, Chlamydia, and tuberculosis. We questioned whether this family of evolutionarily-conserved proteins had a significant role in periodontal disease. ${ }^{7}$ These proteins, defined in part by the molecular size of their prototype 
members, have significant roles in normal cellular function as molecular chaperones 8,9 and defense against environmental stresses. ${ }^{10}$ They have been shown to have an important role in inflammatory mechanisms, ${ }^{11}$ autoimmune diseases, ${ }^{12-14}$ and atherosclerosis. ${ }^{15}$ In addition, these proteins have been shown to be the immunodominant antigens of many microorganisms including periodontal diseaseassociated microorganisms such as Porphyromonas gingivalis ${ }^{16-19}$ and Actinobacillus actinomycetemcomitans. ${ }^{20-21}$ Because these proteins have been so highly conserved in evolution we felt that they might be used to assess "basal" or "generic" immunity in a variety of disease processes and would not necessarily be specifically linked to a single microorganism. In the present study, we have employed a panel of purified stress proteins and measured serum IgG antibody levels to them using particle concentration fluorescence immunoassay. These data were then compared to clinical measurements of periodontal disease and colonization by microorganisms commonly associated with periodontal disease. The specimens used in this study were derived from an Amish and non-Amish population examined in an earlier study. 22,23

\section{MATERIALS AND METHODS}

\section{Study Population}

This study focused on the Amish and non-Amish living in rural southeastern Michigan. The clinical data and archived specimens collected from a total of 198 Amish $(n=145)$ and non-Amish $(n=53)$ specimens were used. This represented all of the subjects recruited during the first year of an earlier study.22,23 The results of that study indicated that the Amish did not present as a unique population that could be distinguished from the non-Amish. This study was performed using clinical findings and specimens archived from that study. Participation rate for these subjects was approximately $77 \%$. Ages ranged from 18 to 79 years; 49\% (97) males and 51\% (101) females. Since the non-significant differences found in clinical measurements between the Amish and non-Amish were attributable to oral hygiene practice, later analyses treated all subjects as a single population. Subsequent regression modeling confirmed this assumption. The oral disease in this population is consistent with age-associated chronic adult periodontitis with no evidence of early-onset periodontal diseases.

\section{Clinical Measurements}

One dentist conducted examinations of all existing teeth with the exception of third molars using the Silness and Löe plaque index, ${ }^{24}$ Löe and Silness gingival index, ${ }^{25}$ and modified Ramfjord's periodontal disease index for probing depth and attachment loss measurements. ${ }^{26}$ Loss of periodontal attachment and pocket depth were measured to the nearest millimeter using a periodontal probe. Measurements were taken from 6 sites of each tooth: disto-buccal, midbuccal, mesio-buccal, disto-lingual, mid-lingual, and mesio-lingual.

\section{Blood Collection}

Blood samples were collected in $10 \mathrm{ml}$ vacuum tubes without anticoagulant. After clotting, the tubes were centrifuged and the serum frozen at $-80^{\circ} \mathrm{C}$.

\section{Plaque Specimens}

Plaque samples were collected from the 6 most periodontally diseased sites based on probing depth and/or inflammation. These 6 samples were then pooled. This approach allowed us to assess the flora representative of the most diseased sites in each subject. Supragingival plaque about the most diseased sites was removed with a sterile curet and discarded. A second sterile curet was introduced into the sulcus or periodontal pocket and extended as far apically as possible. The root surface was then sampled with a curet and the adherent plaque on the scaler tip transferred to a screw-capped cryotube containing $0.5 \mathrm{ml}$ phosphate buffered saline (PBS; $0.05 \mathrm{M}$ sodium phosphate, $0.15 \mathrm{M} \mathrm{NaCl}$, pH 7.4) with $0.5 \%$ formaldehyde, EDTA (2 mM), PMSF (1.0 mM), pepstatin A (0.1 mM) and leupeptin $(0.5 \mathrm{mg} / \mathrm{l})$. Sample vials were stored at $-20^{\circ}$ until assayed.

\section{Slot Immunoblot Assay (SIB)}

The SIB was performed as previously described. ${ }^{27}$ The samples were ultrasonicated $\$$ ( 3 to 5 seconds; $60 \%$ power) to disrupt aggregates of plaque particles. Nitrocellulose sheets"l were soaked for 15 minutes in TBS (0.05 M NaCl, $10.0 \mathrm{mM}$ Tris- $\mathrm{HCl}, 10.0 \mathrm{mM}$ Trisbase, $\mathrm{pH}$ 7.4) and inserted into the slot blot manifold. " Standards (pure cultures) or undiluted plaque samples were applied $(10 \mu \mathrm{l})$ to each well of the slot blot manifold which was then evacuated with gentle application of vacuum. The nitrocellulose membrane was then removed from the manifold for further processing. All subsequent incubations were performed on a rocking table ${ }^{\#}$ at room temperature.

Microbial detection and quantitation was performed after first immersing the nitrocellulose membrane in TBS containing $0.5 \%$ non-fat dried milk** for 30 to 60 minutes to block unoccupied binding sites on the nitrocellulose membrane. The appropriate antibacterial antibody, diluted in TBS-Tween 20 (TBS-T; 0.05\% Tween 20 ) containing $0.5 \%$ milk proteins* * was

$\S$ Kontes Instruments.

II BA-85, Schleicher E Schuell, Keene, NH.

I Minifold II, Scheicher \& Schuell.

\# Hoefer Scientific Co., San Francisco, CA

** BLOTTO, Kirkegaard E Perry Laboratories, Gaithersburg, MD. 
Table I.

\section{Clinical Description of Subject Population}

\begin{tabular}{lcc}
\hline Clinical Measurement & Mean \pm S.D. & $\begin{array}{c}\text { Range of Means by Subject } \\
\text { (minimum-maximum) }\end{array}$ \\
\hline Plaque index & $0.87 \pm 0.59$ & $0.26-1.93$ \\
Gingival index & $0.83 \pm 0.63$ & $0.17-2.32$ \\
Probing depth $(\mathrm{mm})$ & $2.65 \pm 0.55$ & $1.34-4.38$ \\
Attachment loss $(\mathrm{mm})$ & $1.24 \pm 0.81$ & $0.23-4.66$ \\
\hline
\end{tabular}

FITC-labeled anti-human IgG in the absence of 1:100 dilution of patient serum to control for non-specific binding. Preliminary studies indicated that a 1:100 serum dilution was optimal, resulting in detection of all sample fluorescence at the same gain setting (gain $=25$ ) in the linear range of the machine. The relative amounts of fluorescence in each well was used as a semiquantitative representation of the amount of antibody present in the sample.

\section{Statistical Analysis}

The relationships between specific indices of periodontal disease, antibody level, and coloapplied and allowed to incubate for 1 hour. Following three 5-minute washes in TBS-T, goat anti-rabbit IgG conjugated to alkaline phosphatase ${ }^{\dagger \dagger}$ diluted in TBS$\mathrm{T}$ containing $0.5 \%$ milk proteins was applied and incubated for 1 hour. After all washes, the BCIP-NBT substrate solution ${ }^{\ddagger}$ was applied and color development allowed to proceed to its maximum. Absorbed polyclonal antisera prepared in rabbits specific for $P$. gingivalis, $A$. actinomycetemcomitans, T. denticola, and $B$. forsythus were used in the SIB assay. The specificity of these antisera has been previously described. 28,29

\section{Particle Concentration Fluorescence Immunoassay (PCFIA)}

PCFIA was performed as described by Shelburne et al. ${ }^{30}$ Stress proteins used in these studies were limited to those that were commercially available since periodontal pathogen-specific stress proteins were either not available or were available in amounts insufficient for screening purposes. The initial step in the performance of PCFIA was the preparation of antigencoupled latex particles. Briefly, $100 \mu \mathrm{g}$ of the specific stress protein (human Hsp70, human Hsp90, E. coli DnaK, and E. coli GroEL) was diluted to $10 \mathrm{ml}$ in $0.1 \mathrm{M}$ sodium phosphate ( $\mathrm{pH} \mathrm{7.0)}$ and combined with $10 \mathrm{mg}$ of carbodiimide§§ and $1 \mathrm{ml}$ of carboxyl latex particles ${ }^{\prime l l}$ $(0.8 \mu \mathrm{M}, 5 \% \mathrm{w} / \mathrm{v}$,$) and incubated for 1$ to 2 hours at $37^{\circ} \mathrm{C}$ with rotation. The coated particles were washed 3 times in $0.1 \mathrm{M}$ sodium phosphate and resuspended in $20 \mathrm{ml}$ of PBS $+0.2 \%$ sterile milk proteins. Twenty $\mu \mathrm{l}$ of the coated particles were dispensed into each well of 96 well filtration plates. III Sera were diluted 1:100 in PBS $+0.2 \%$ sterile milk proteins, added to wells $(20 \mu \mathrm{l} /$ well) in triplicate, and incubated 30 minutes at room temperature. The particles were then washed and $20 \mu \mathrm{l}$ of FITC labeled anti-human IgG (various vendors) added. After an additional 15-minute incubation the particles were washed 3 times and the fluorescence of the labeled anti-human IgG captured by the solid phase was measured by epifluorimetry and expressed in relative fluorescence units (RFU). Controls included pooled human serum to control for inter-plate variability and nization of subgingival dental plaque were assessed by ANOVA. Fisher's method for multiple comparisons was used for pair-wise comparison of the groups. The contribution of these variables to stress protein antibody concentration, as well as those of age, gender, and Amish/non-Amish status, was assessed in multiple and stepwise regression models. "fll Results with $P$ $\leq 0.05$ were considered significant.

\section{RESULTS}

\section{Clinical Measurements}

A complete description of the clinical status of this population is available in earlier reports. ${ }^{22,23} \mathrm{An}$ overview of the clinical status of the subjects is provided in Table 1. All analyses described below used full mouth means for all clinical measurement (probing depth, attachment loss, and gingival index).

\section{Colonization and Clinical Measurements}

The relationship between colonization and the clinical measurements was determined by ANOVA. As shown in Table 2, when probing depth was examined, increasing probing depth was associated with increased colonization by all species tested. In all cases but $A$. actinomycetemcomitans, these increases were significant. We observed no statistically significant relationships when gingival index or attachment loss were evaluated.

\section{Anti-Stress Protein Serum Antibody Concentrations and Clinical Measurements}

Subjects were stratified into 3 groups $(0,1$, and 2) based on their mean whole mouth gingival index $(0=0$ to $<0.5 ; 1=0.5$ to $<1.5 ; 2=>1.5$ ). The anti-stress protein antibody concentrations in these 3 groups were then compared by ANOVA. As shown in Figure 1B, a consistent trend is noted. Gingival index strata of 0 and 1 were associated with higher antibody concentrations, while scores greater than 1 (significant amounts of gin-

$\dagger \dagger$ Bio-Rad Laboratories, Hercules, CA.

$\neq \ddagger$ Kirkegaard \& Perry Laboratories.

$\S \S$ Sigma Chemical Co., St. Louis, MO.

III Epicon, IDEXX, Westbrook, ME.

ขा Stat-View, SAS Institute, Cary, NC. 
givitis) were associated with lower levels of antibody. In the case of anti-Hsp90 and anti-GroEL antibodies, the concentrations were significantly $(P<0.05)$ lower in the stratum 2 category compared to the 0 category. For the other stress proteins, while trends were apparent, the differences were not within the 95\% confidence level.

A similar pattern is seen when examining the relationship between mean probing depth and anti-stress protein antibody concentration (Fig. 1A). The highest antibody concentrations were found in subjects in the $2 \mathrm{~mm}$ and $3 \mathrm{~mm}$ strata. Subjects who have mean probing depths greater than $3 \mathrm{~mm}$ ( $3 \mathrm{~mm}$ stratum) have significantly $(P<0.05)$ reduced antibody concentrations for all stress proteins, except Hsp70. Figure 1C shows the same relationships for attachment loss. Again, the same trend is obvious and is significant $(P<0.05)$ for Hsp90 and DnaK.

We then examined the relationship between the presence of anti-stress protein antibodies and colonization of the subgingival plaque by specific periodontal disease-associated microorganisms (Fig. 2). In this figure, we show the relationship between the antibody concentrations and 3 levels of colonization as measured by the slot immunoblot assay. These levels (low, moderate, and high) correspond to $<0.2 \times 10^{5}$, 0.2 to $3 \times 10^{5}$, and $>3 \times 10^{5}$ microorganisms ${ }^{27}$ in the undiluted plaque sample assessed in the assay. As shown in Figure 2A, there is an association between colonization and anti-Hsp90 concentration. In all cases, there is a significantly $(P<0.05)$ lower antiHsp90 concentration associated with the lowest level of colonization regardless of the bacterial species examined. In all cases, with the exception of A. actinomycetemcomitans, the highest antibody concentration is associated with the highest level of colonization.

There does not appear to be any significant association in the relationship between the anti-Hsp70 concentrations and colonization. There is an apparent association with $B$. forsythus colonization; however, it is not statistically significant. This is also the case when the associations between anti-GroEL antibodies and colonization are examined (Fig. 2C). In the cases of $A$. actinomycetemcomitans and $B$. forsythus while the antibody concentrations appear to be lower at the highest level of colonization, they are not statistically significant.

Finally, significant $(P<0.05)$ relationships between the anti-DnaK antibody concentrations and colonization by $P$. gingivalis and $T$. denticola are evident in
Figure 2D. Though not significant, A. actinomycetemcomitans reveals a trend with anti-DnaK antibodies similar to that observed for the other three antibodies. In all cases, the antibody concentration at the highest level of colonization is lower than the preceding concentration. Similarly, B. forsythus reveals this pattern with anti-DnaK and anti-GroEL antibodies.

\section{Regression Modeling of Anti-Stress Protein Antibodies}

In order to begin to understand the variables that contribute to the anti-stress protein antibody concentrations, we used step-wise and multiple regression models which examined the contribution of age, gender, Amish/non-Amish status, gingival index, probing depth, attachment loss, calculus index, plaque index, and microbial colonization. In the case of the Hsp90 antibodies, 2 variables were found to have significant contributions to the antibody concentration having a $\mathrm{R}=0.293$ and $P<0.0002$. These variables were probing depth (PD) and $P$. gingivalis concentration (PG). The equation derived from the regression model was $y=12558-2070 *$ PD $+1842 *$ PG. This confirmed the inverse relationship with probing depth and the positive relationship with colonization by $P$. gingivalis. Attempts to model the other stress protein antibodies were not successful.

\section{DISCUSSION}

There has been a considerable focus on stress proteins in current infectious disease research due to the observations that these protein families are immunodominant antigens of many human pathogens. ${ }^{7}$ As a result we felt that it was timely to assess the association between immunity to common stress proteins and one of the most common microbial-mediated diseases in humans, periodontal disease. We selected 4 common, commercially available stress proteins, 2 human and 


\section{Gingival Index}

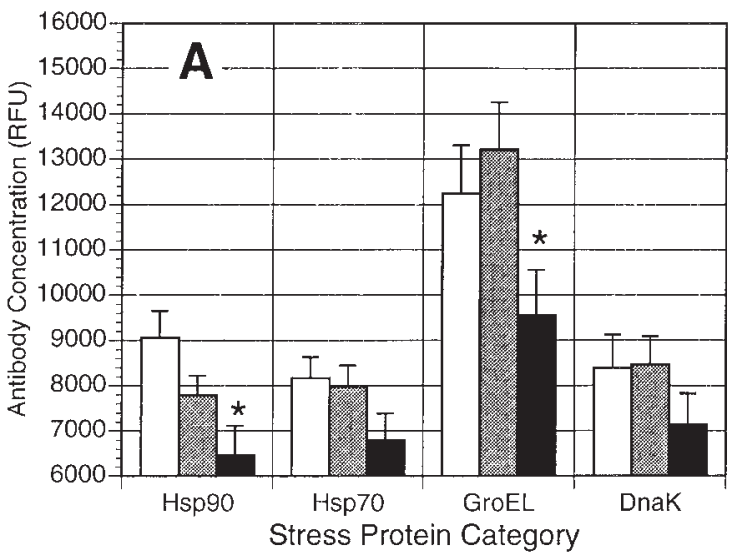

Attachment Loss

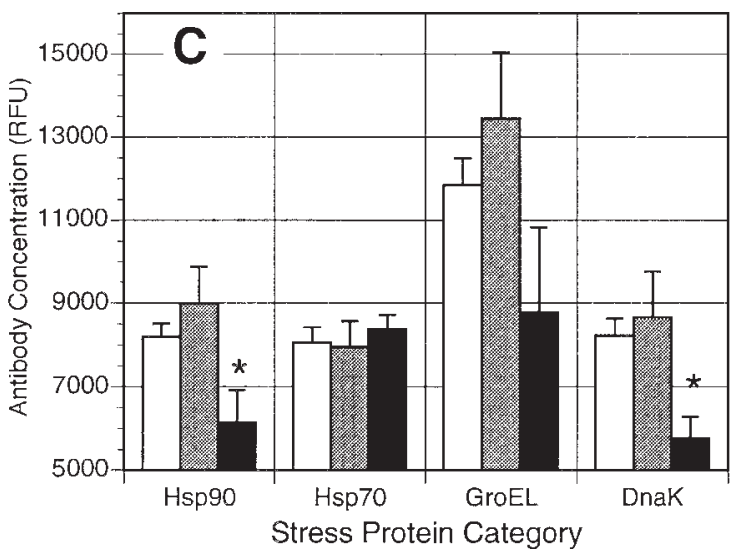

2 microbial in origin. Human Hsp70 and Hsp90 were used because they are amongst the most common of the stress protein families in eukaryotic systems. ${ }^{10}$ Since there is evidence in studies of Candida albicans that conserved Hsp90 epitopes, shared between humans and Candida, were responsible for protection against systemic candidiasis, we felt that evaluating the association between anti-human Hsp antibodies and oral disease might be informative. ${ }^{31}$ We chose GroEL and DnaK (Hsp65 and Hsp70) since they represented the most highly conserved stress proteins of microbial origin. ${ }^{10}$

The clinical specimens were derived from a patient population that was from an earlier study of an Amish community. ${ }^{22,23}$ Based on an evaluation of the oral health of this population and comparisons with nonAmish living in the same geographical location, we felt that, with the exception of a lack of routine oral

\section{Probing Depth}

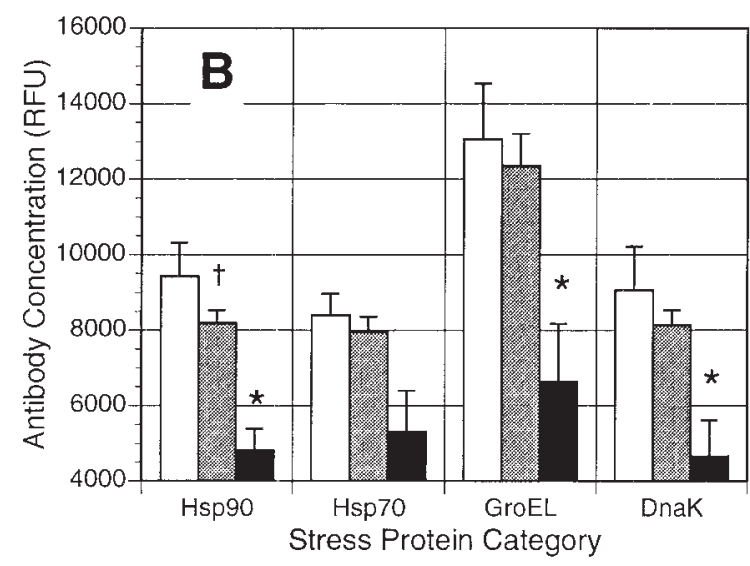

Figure I.

Relationship between clinical measurements and serum anti-stress protein antibody concentration. Antibody concentration for each stress protein is expressed in relative fluorescence units (RFU) as determined by particle concentration fluorescence immunoassay (PCFIA). Error bars indicate standard error of the mean for the antibody concentration in each clinical category. Statistical significance of antibody titers ( $P \leq 0.05)$ : *significant differences between lowest and highest clinical stratum;

tsignificant differences between middle and highest clinical stratum. A. Mean gingival index; subjects were stratified into 3 groups (0, I, and 2; white, gray, and black bars, respectively) based on their mean whole mouth gingival index $(0=0$ to $<0.5 ; 1=0.5$ to $<1.5 ; 2=>1.5)$. Sample sizes for categories 0,1 , and 2 were 87,66 , and 45 , respectively. B. Mean probing depth; subjects were stratified into 3 groups $(2 \mathrm{~mm}$, $3 \mathrm{~mm}$, and $4 \mathrm{~mm}$; white, gray, black bars, respectively) based on their mean whole mouth probing depths $(2 \mathrm{~mm},<2.5 \mathrm{~mm} ; 3 \mathrm{~mm}, \geq 2.5$ and $<3.5 ; 4 \mathrm{~mm}, \geq 3.5 \mathrm{~mm}$ ). Sample sizes for categories $2 \mathrm{~mm}, 3 \mathrm{~mm}$, and $3 \mathrm{~mm}$ were 68,72 , and 58, respectively.

C. Mean attachment loss; subjects were stratified into 3 groups $(2 \mathrm{~mm}$, $3 \mathrm{~mm}$ and $4 \mathrm{~mm}$; white, gray, black bars, respectively) based on their mean whole mouth attachment losses $(2 \mathrm{~mm},<2.5 \mathrm{~mm} ; 3 \mathrm{~mm}, \geq 2.5$ and $<3.5 \mathrm{~mm}, \geq 3.5 \mathrm{~mm}$ ). Sample sizes for categories $2 \mathrm{~mm}, 3 \mathrm{~mm}$ and $4 \mathrm{~mm}$ were 86,79 , and 33 , respectively. hygiene, there were no significant differences between the Amish and the non-Amish in this area. While this is a randomly-selected population with few members presenting with advanced periodontitis, there is a clear relationship between disease severity (probing depth) and level of colonization by putative periodontal pathogens.

Our studies also demonstrated a clear relationship between the serum concentration of 3 of the 4 specific anti-stress protein antibodies and clinical measures of periodontal disease. The serum antibodies (IgG) were highest when the subjects have the best oral health. This was most obvious when the mean probing depths are examined. It was interesting that throughout these analyses, while the antibodies reactive with human Hsp70 tended to follow the same trends, they showed the least statistically significant relationships. However, its microbial homologue, DnaK, demonstrated consid- 

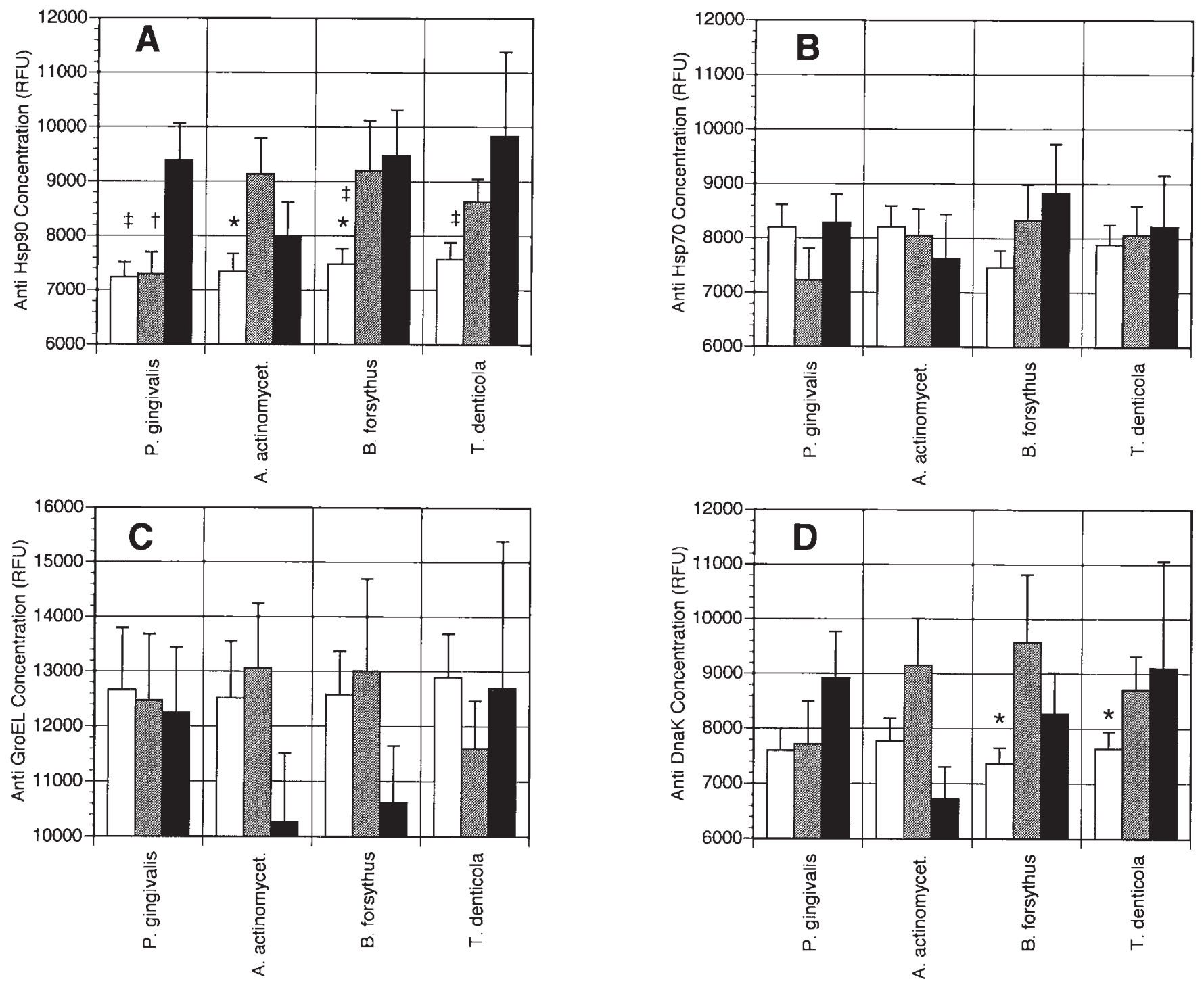

Figure 2.

Relationship between serum anti-stress protein antibody concentration and colonization. Antibody concentration for each stress protein is expressed in relative fluorescence units (RFU) as determined by particle concentration fluorescence immunoassay (PCFIA). Error bars indicate standard error of the mean for the antibody concentration in each colonization category. Colonization by selected microorganisms is expressed as low (SIB score $=0)$, moderate (SIB score $=$ 1) and high (SIB score $=2+3$ ) (white, gray, and black bars, respectively). Statistical significance of antibody titers (P $\leq 0.05)$ : *for significant differences between low and moderate colonization; tfor significant differences between low and high colonization; and for significant differences between moderate and high colonization. A) Anti-Hsp90 antibodies; B) Anti-Hsp70 antibodies; C) Anti-GroEL antibodies; D) Anti-DnaK antibodies. Sample sizes for low, medium, and high colonization categories by microorganism are as follows: P. gingivalis, 43, 44, I I I (L,M,H, respectively); A. actinomycetemcomitans, 5 I, I I 0, 37; B.

forsythus, 91, 74, 33; and T. denticola, 83, 71, 44.

erable disease-associated differences. This would suggest that even with the considerable homology within the Hsp70 family, the epitopes that are being recognized immunologically are probably those which are not evolutionarily conserved. In contrast, a very significant inverse relationship between disease and human Hsp90 antibody concentration probably indicates that highly conserved epitopes are being recognized. While we did not possess purified microbial Hsp90 protein at the time of this study, investigations in progress in our laboratory demonstrate a high degree of cross-reactivity between human Hsp90 and the Hsp90 homologues of $P$. gingivalis and $A$. actinomycetemcomitans based on antibody reactivity (unpublished data). Similarly, serum IgG antibody concentrations reactive with GroEL followed similar trends. Mean gingival indices and attachment losses also followed these trends.

Our observations are somewhat similar of a report by Schett et al. ${ }^{32}$ They examined anti-Hsp65 antibodies in sera (IgG) and found that the antibody concen- 
trations were highest in periodontitis. This conflicts with our observations that in periodontitis the anti-Hsp antibody levels are lowest. A possible explanation for this discrepancy is not immediately obvious; however, specific differences between our studies exist. There is a considerable difference in sample size between the studies. In their study, a total of 23 subjects were divided into 3 groups. There are also differences between the antigens tested. We tested only $E$. coli GroEL and Schett et al. ${ }^{32}$ reported serum (IgG) data only for recombinant mycobacterial Hsp65. Finally, and perhaps the most important difference is that in our random population, we do not have the severe periodontitis that is observed in the Schett groups. And as a result, our most diseased group is considerably healthier than theirs and resembles their intermediate (gingivitis) disease.

Our study also examined the association between colonization by specific microbial species and antistress protein antibodies. We found no significant associations between anti-GroEL or anti-Hsp70 concentrations and colonization by the specific microorganisms in our test panel. Again, we found this interesting since relationships between antibody concentration and colonization were observed with antiDnaK antibodies, an Hsp70 homologue, again indicating that, in this case, the non-conserved (antibacterial) epitopes are probably more important. However, we observed the most significant associations between the anti-Hsp90 concentrations and microbial colonization. Since the antigen used to test for anti-Hsp90 antibodies is derived from human sources, the antibodies detected most likely represent those directed against conserved epitopes, rather than species-specific or anti-bacterial. In all cases, except for $A$. actinomycetemcomitans, there appeared to be a significant association between increased colonization and anti-Hsp90 antibody concentration.

Statistical modeling clearly demonstrated the inverse relationship with probing depth and the positive relationship with colonization. The model very clearly defines the relationship between $P$. gingivalis and Hsp90. The most surprising observation is the inverse relationship between the antibody concentrations and oral disease. Most prior studies of humoral immunity to periodontal disease-associated microorganisms and oral health have shown a positive correlation. ${ }^{1}$ Thus, the present observations may actually be identifying the presence of protective antibodies, rather than simply presence of the microorganism in the gingival sulcus. If these are, in fact, protective antibodies, the next critical step will be to localize the antigens using these antibodies in order to help assess the role of the antigen in the virulence of the microorganism. It will be critical to determine if the stress proteins play a role in adherence and colonization, or if they interfere with normal mammalian cell function once the microorganism has established its infection.

Homologues of specific stress protein families have been demonstrated to be present in oral bacteria, including F. nucleatum, P. intermedia, P. nigrescens, $P$. melaninogenica, A. actinomycetemcomitans, and P. gingivalis. Hsp60/GroEL and Hsp70/DnaK homologues have been detected using cross-reactive antibodies against prototype members. ${ }^{16,19,20,33,34}$ Homologues of DnaK, GroEL, and groESL have been cloned and sequenced from $P$. gingivalis and $A$. actinomycetemcomitans. ${ }^{17,18,20,33,35,36}$ In addition, the hsp90 gene homologue of A. actinomycetemcomitans was "accidentally" cloned by Winston et al. ${ }^{37}$

There have been numerous suggestions that the Hsp of pathogens may play a role in their virulence ${ }^{38,39}$ Studies by Matthews and Burnie ${ }^{31}$ demonstrate that immunity to Candida albicans Hsp90 is important for survival from systemic candidiasis. Other studies have shown that the normally nonpathogenic $S$. cerevisiae can be made pathogenic by causing an over-expression of the hsp90 gene. ${ }^{40}$ Mutations in an hsp gene ( $h$ trA) in Salmonella typhimurium result in reduced virulence. Finally, reports by Kirby et al. have shown that the potent bone-resorbing mediator of A. actinomycetemcomitans is homologous to GroEL. ${ }^{41}$ These findings support the importance of understanding the role of stress proteins in microbial virulence and the function of the host response in blocking these effects.

Our studies employing stress protein homologues reveal that there is considerable conservation of Hsp epitopes with periodontal disease-associated microorganisms. Clearly, since we were able to demonstrate associations using purified human Hsp, autoimmunity (as opposed to autoimmune disease) may serve an important function in host defense in periodontal disease. This is not a novel observation and has been proposed by other investigators studying the role of host immunity to stress proteins. ${ }^{34}$

The relationship between anti-Hsp90 immunity, oral health, and colonization is reminiscent of the observations by Matthews and Burnie ${ }^{31}$ in their studies of systemic candidiasis. In interpreting our findings, it should be clear that we are not using antigens derived from any of the periodontal disease-associated microorganisms in our panel. The serum antibodies and the associations that we have described are likely to reflect the contributions of specific antigenic epitopes that have been conserved through evolution due to their importance to the inherent function of the stress protein. We would expect that if we had employed stress proteins derived from our microbial panel, significantly different results would have been obtained. In that case, we would predict that the predominant serum antibody response would be against the species-specific epitopes 
and would probably reflect infection "history" rather than protective antibodies. Our laboratory is currently cloning the Hsp90 homologue of $P$. gingivalis and once complete we will be able to address issues related to homology between human and P. gingivalis Hsp90 and the epitopes that are involved in this apparent protective response. Once sufficient quantities of purified or recombinant Hsps of periodontal disease-associated microorganisms are available we will be able to begin to address the importance of immunity to conserved versus microbe-specific epitopes. Since Matthews and Burnie $^{31}$ demonstrated that protection could be attributed to the recognition of a specific conserved epitope, it may be possible to establish a genetic basis for resistance to $P$. gingivalis (or other species) colonization based on the ability to recognize a specific epitope. Further elucidation of this model is dependent on evaluating the immunity to specific Hsp90 epitopes.

\section{ACKNOWLEDGMENTS}

The authors acknowledge the expert technical assistance provided by Mrs. Christine A. Binsfeld in the performance of the PCFIA analysis. Mr. Shelburne is a Research Specialist in the BioMaterials Technology Center of 3M. This study was supported by U.S. Public Service Grants DE 11117, DE08571, and DE06998.

\section{REFERENCES}

1. Ebersole JL. Systemic humoral immune responses in periodontal disease. Crit Rev Oral Biol Med 1990;1: 283-331.

2. Boutsl EA, Koseki T, Nishihara T, Ishikawa I. Characterization of the immunodominant antigens of Porphyromonas gingivalis 381 in high-responder patients. Oral Microbiol Immunol 1996;11:236-241.

3. Wilson M, Reddi K, Henderson B. Cytokine-inducing components of periodontopathogenic bacteria. J Periodont Res 1996;31:393-407.

4. Wilson M. Biological activities of lipopolysaccharides from oral bacteria and their relevance to the pathogenesis of chronic periodontitis. Sci Prog 1995;78:19-34.

5. Yoshimura F, Sugano T, Kawanami M, Kato H, Suzuki T. Detection of specific antibodies against fimbriae and membrane proteins from the oral anaerobe Bacteroides gingivalis in patients with periodontal diseases. Microbiol Immunol 1987;31:935-941.

6. Daly CG, Seymour GJ, Kieser JB. Bacterial endotoxin: a role in chronic inflammatory periodontal disease? J Oral Pathol 1980;9:1-15.

7. Shinnick TM. Heat shock proteins as antigens of bacterial and parasitic pathogens. Curr Top Microbiol Immunol 1991;167:145-160.

8. Craig EA, Gambill BD, Nelson RJ. Heat shock proteins: molecular chaperones of protein biogenesis. Microbiol Rev 1993;57:402-414.

9. Hendrick JP, Hartl FU. Molecular chaperone functions of heat-shock proteins. Ann Rev Biochem 1993;62: 349-384.

10. Lindquist S. The heat-shock response. Ann Rev Biochem 1986;55:1151-1191.

11. Polla BS. A role for heat shock proteins in inflammation? Immunol Today 1988;9:134-137.
12. Minota S, Cameron B, Welch WJ, Winfield JB. Autoantibodies to the constitutive 73-kD member of the hsp70 family of heat shock proteins in systemic lupus erythematosus. J Exp Med 1988;168:1475-1480.

13. Minota S, Koyasu S, Yahara I, Winfield J. Autoantibodies to the heat-shock protein hsp90 in systemic lupus erythematosus. J Clin Invest 1988;81:106-109.

14. Winfield J, Jarjour W, Minota S. Stress protein autoantibodies and the expression of stress proteins on the surface of human gamma-delta cells and other cells of the immune system. Chem Immunol 1992;53:47-60.

15. Kleindienst R, Schett G, Amberger A, et al. Atherosclerosis as an autoimmune condition. Isr J Med Sci 1995;31:596-599.

16. Lu B, McBride BC. Stress response of Porphyromonas gingivalis. Oral Microbiol Immunol 1994;9:166-173.

17. Maeda H, Miyamoto M, Hongyo H, Nagai A, Kurihara H, Murayama Y. Heat shock protein 60 (GroEL) from Porphyromonas gingivalis: molecular cloning and sequence analysis of its gene and purification of the recombinant protein. FEMS Microbiol Lett 1994;119: 129-135.

18. Maeda H, Miyamoto M, Hongyo H, Nagai A, Kurihara H, Muryama Y. Heat shock protein 60 (GroEL) from Porphyromonas gingivalis: molecular cloning and sequence analysis of its gene and purification of the recombinant protein. FEMS Microbiol Lett 1994:15; 124:121-112.

19. Vayssier C, Mayrand D, Grenier D. Detection of stress proteins in Porphyromonas gingivalis and other oral bacteria by western immunoblotting analysis. FEMS Microbiol Lett 1994;121:303-307.

20. Nakano Y, Inai Y, Yamashita Y, et al. Molecular and immunological characterization of a $64-\mathrm{kDa}$ protein of Actinobacillus actinomycetemcomitans. Oral Microbiol Immunol 1995;10:151-159.

21. Lokensgard I, Bakken V, Schenck K. Heat shock response in Actinobacillus actinomycetemcomitans. FEMS Immunol Med Microbiol 1994;8:321-328.

22. Bagramian RA, Farghaly MM, Lopatin D, Sowers M, Syed SA, Pomerville JL. A comparison of periodontal disease among rural Amish and non-Amish adults. J Clin Periodontol 1994;21:386-390.

23. Bagramian RA, Farghaly MM, Lopatin D, Sowers M, Syed, SA, Pomerville JL. Periodontal disease in an Amish population. J Clin Periodontol 1993;20:269-272.

24. Silness J, Löe H. Periodontal disease in pregnancy. (II) Correlation between oral hygiene and periodontal condition. Acta Odontol Scand 1964;22:121-135.

25. Löe H, Silness J. Periodontal disease in pregnancy. (I) Prevalence and severity. Acta Odontol Scand 1963;21: 533-551.

26. Ramfjord SP. The periodontal disease index (PDI). J Periodontol 1967;38(Suppl.):602-610.

27. Van Poperin N, Lopatin DE. Slot immunoblot assay for detection and quantitation of periodontal diseaseassociated microorganisms in dental plaque. J Clin Microbiol 1991;29:2554-2558.

28. Loesche WJ, Lopatin DE, Giordano J, Alcoforado G, Hujoel P. Comparison of the benzoyl-DL-argininenaphthylamide (BANA) test, DNA probes, and immunological reagents for ability to detect anaerobic periodontal infections due to Porphyromonas gingivalis, Treponema denticola, and Bacteroides forsythus. J Clin Microbiol 1992;30:427-433.

29. Loesche WJ, Lopatin DE, Stoll J, Van Poperin N, Hujoel PP. Comparison of various detection methods for 
periodontopathic bacteria: can culture be considered the primary reference standard? J Clin Microbiol 1992;30: 418-426.

30. Shelburne CE, Sandberg GP, Binsfeld CA, Wolff LF, Curry RA. Monoclonal antibodies to lipopolysaccharide of four oral bacteria associated with periodontal disease. J Periodont Res 1993;28:1-9.

31. Matthews R, Burnie J. The role of hsp90 in fungal infection. Immunol Today 1992;13:345-348.

32. Schett G, Metzler B, Kleindienst R, et al. Salivary antihsp65 antibodies as a diagnostic marker for gingivitis and a possible link to atherosclerosis. Int Arch Allergy Immun 1997;114:246-250.

33. Koga T, Kusuzaki T, Asakawa H, Senpuku H, Nishihara T, Noguchi T. The 64-kilodalton GroEL-like protein of Actinobacillus actinomycetemcomitans. J Periodontal Res 1993;28:475-477.

34. Ando T, Kato T, Ishihara K, Ogiuchi H, Okuda K. Heat shock proteins in the human periodontal disease process. Microbiol Immunol 1995;39:321-327.

35. Hotokezaka H, Ohara N, Hayashida H, et al. Transcriptional analysis of the groESL operon from Porphyromonas gingivalis. Oral Microbiol Immunol 1997;12: 236-239.

36. Yoshida A, Nakano Y, Yamashita Y, Yu H, Ohishi M, Koga $\mathrm{T}$. Isolation and characterization of the dnaKJ operon from Actinobacillus actinomycetemcomitans. DNA Sequence 1997;8:93-98.

37. Winston JL, Toth SI, Roe BA, Dyer DW. Cloning and characterization of the Actinobacillus actinomycetemcomitans gene encoding a heat-shock protein 90 homologue. Gene 1996;179:199-204.

38. Lathigra RB, Butcher PD, Garb, TR, Young DB. Heat shock proteins as virulence factors of pathogens. Curr Top Microbiol Immunol 1991;167:125-143.
39. Fernandez RC, Logan SM, Lee, SH, Hoffman PS. Elevated levels of Legionella pneumophila stress protein Hsp60 early in infection of human monocytes and L929 cells correlate with virulence. Infect Immun 1996;64: 1968-1976.

40. Hodgetts S, Matthews R, Morrissey G, Mitsutake K, Piper $\mathrm{P}$, Burnie J. Over-expression of Saccharomyces cerevisiae hsp90 enhances the virulence of this yeast in mice. FEMS Immunol Med Microbiol 1996;16:229-234.

41. Kirby AC, Meghji S, Nair SP, et al. The potent boneresorbing mediator of Actinobacillus actinomycetemcomitans is homologous to the molecular chaperone GroEL. J Clin Invest 1995;96:1185-1194.

Send reprint requests to: Dr. Dennis E. Lopatin, Department of Biologic and Materials Sciences, School of Dentistry, University of Michigan, 1011 North University Ave., Ann Arbor, MI 48109-1078. Fax: 734/764-2425.

Accepted for publication February 8, 1999. 